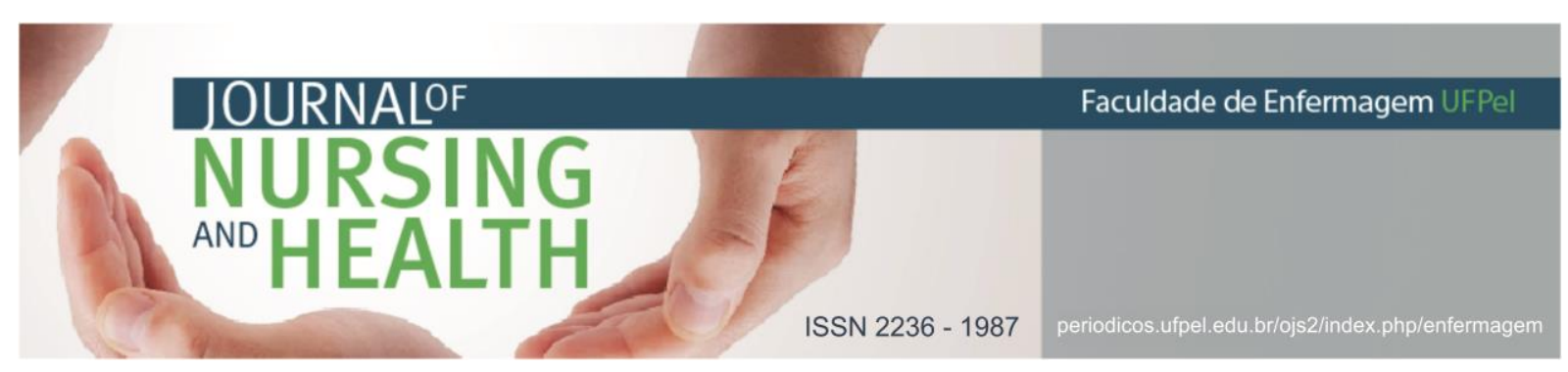

RELATO DE EXPERIÊNCIA

\title{
Movilidad internacional en enfermería: oportunidad de ampliar la visión del mundo y agregar conocimientos
}

\author{
Mobilidade internacional em enfermagem: oportunidade de ampliar a visão do mundo \\ e agregar conhecimentos
}

\author{
International mobility in nursing: opportunity to expand the world view and add \\ knowledge
}

Couto, Daniela Sanches ${ }^{1}$; Pereira, Queli Lisiane Castro

Como citar este artigo: Couto DS, Pereira QLC. Movilidad internacional en enfermería: oportunidad de ampliar la vision del mundo y agregar conocimientos. J nurs health. 2018;8(2):e188204

\section{RESUMEN}

Objetivo: relatar la experiencia de la movilidad académica internacional en México a través del Programa Internacional de Estudiantes Brasil-México, durante la graduación de enfermería. Métodos: este es un relato de experiencia académica en México, en la Facultad de Enfermería y Obstetricia, Universidad Juárez del Estado de Durango hecho posible por la Universidad Federal de Mato Grosso, Brasil, de agosto a diciembre de 2017. Resultados: el intercambio de experiencias que se produjo dentro de un semestre, permitió la comprensión de nuevos conocimientos y el desarrollo de vínculos estudiantiles. Consideraciones finales: en las negociaciones se espera estimular la movilidad internacional en cuenta su importancia para actualidad y como herramienta para la formación de profesionales listos para las diversidades.

Descriptores: Intercambio educacional internacional; Estudiantes de enfermería; Enseñanza.

\section{RESUMO}

Objetivo: relatar a experiencia da mobilidade acadêmica internacional no México por meio do Programa Internacional de Estudiantes Brasil-México, durante a graduación de enfermagem. Métodos: trata-se de um relato da experiência acadêmica no México, na Facultad de Enfermería e Obstetrícia da Universidade Juarez do Estado del Durango, possibilitada pela Universidade Federal de Mato Grosso, Brasil, de agosto a dezembro de 2017. Resultados: a troca de experiências que ocorreu em um semestre permitiu a compreensão de novos conhecimentos e o desenvolvimento de vínculos estudantis. Considerações finais: nas negociações, espera-se estimular a mobilidade internacional, levando em conta sua importância para os assuntos atuais e como ferramenta para a formação de profissionais preparados para as diversidades.

Descritores: Intercâmbio educacional internacional; Estudantes de enfermagem; Ensino.

\footnotetext{
1 Acadêmica do curso de Enfermagem. Universidade Federal do Mato Grosso (UFMT). E-mail: danicouto_2008@hotmail.com http://orcid.org/0000-0003-0767-4000

2 Enfermeira. Doutora em Parasitologia. Universidade Federal do Mato Grosso (UFMT). E-mail: quelilisiane@hotmail.com http://orcid.org/0000-0001-6965-4887
} 


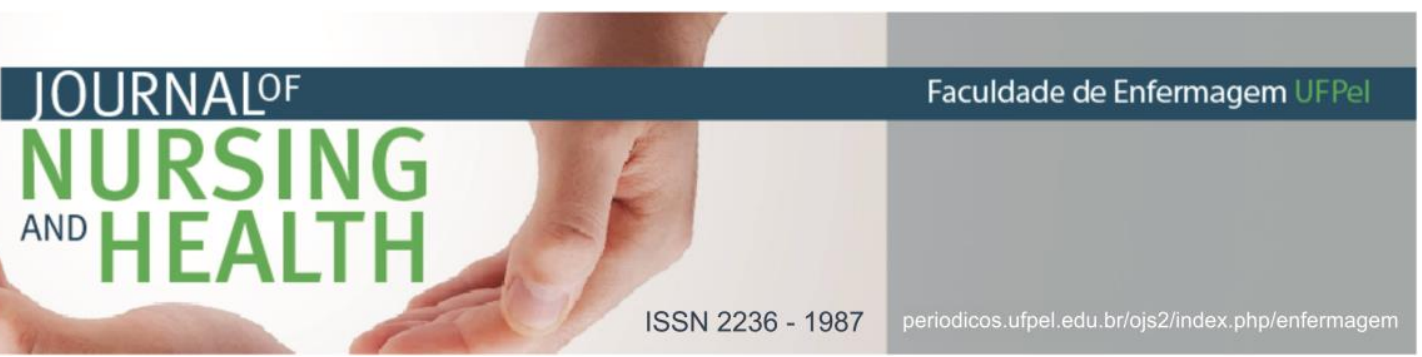

ABSTRACT

Objective: to report the experience of international academic mobility in Mexico through the International Program of Brazil-Mexico Students, during the nursing graduation. Methods: this is an account of academic experience in Mexico, at the Faculty of Nursing and Obstetrics, Universidad Juárez del Estado de Durango made possible by the Federal University of Mato Grosso, Brazil, from August to December 2017. Results: the exchange of experiences that took place within a semester, allowed the understanding of new knowledge and the development of student ties. Final considerations: the negotiations are expected to stimulate international mobility in view of its importance for current events and as a tool for the training of professionals ready for diversities. Descriptors: International educational exchange; Students, nursing; Teaching.

\section{INTRODUCCIÓN}

En las últimas décadas el mundo viene enfrentando grandes cambios socioeconómicos, culturales y tecnológicos, llevando las personas a la necesidad de adaptación a esa sociedad globalizada. A partir de la necesidad de reconstruir los países destruidos después del período de la Segunda Guerra Mundial, en 1945 Europa comenzó la internacionalización en la enseñanza superior que se convirtió en un proceso prioritario para las directrices de educación de los planes de gobierno. ${ }^{1}$

$\mathrm{El}$ proceso

de internacionalización del conocimiento se hace necesario bajo la visión de la globalización, colaborando en la asociación de las cuestiones económicas, sociales, políticas y educativas. ${ }^{2}$ El Gobierno brasileño también tuvo esa intención al promover la expansión de la internacionalización, a través de programas de intercambio y movilidad internacionales en el ámbito universitario. ${ }^{3}$

Dentro de esa visión la internacionalización se ha convertido en un gran campo de conquista por las universidades, ya que compone la principal esfera que estimula el conocimiento. Constituido por pilares que contribuyen al desarrollo económico y al perfeccionamiento de la calidad de vida, a través de la internacionalización. ${ }^{4}$

El contacto con una cultura y de la interacción con personas de diferentes orígenes posibilita una formación crítica y reflexiva sobre las diversas concepciones del mundo. Las universidades presentan una responsabilidad en la construcción de profesionales capacitados para atender las necesidades de una sociedad globalizada. ${ }^{4}$

La experiencia de intercambio añade valores para el crecimiento personal y profesional, por promover contacto con costumbres y culturas, contribuye a la construcción de la autoconfianza, madurez e independencia. ${ }^{3}$ Realizar una modalidad internacional posibilita una nueva mirada sobre el proceso de enseñanza y aprendizaje, en el enfrentamiento de desafíos y en el desarrollo personal. ${ }^{5}$

En el ámbito de la salud, la formación profesional se ha convertido en un punto preocupante para las universidades, por ser la mayor productora de conocimiento utilizando 


\section{ISSN 2236 - 1987}

de la investigación. El mercado profesional, cada vez más, exige aptitud que sobrepasa el conocimiento técnico, incluyendo dominio de otra lengua, conocimiento de las diversidades culturales, facilidad de relacionarse y capacidad de adaptación a las circunstancias que el mundo globalizado impone. ${ }^{3}$

El intercambio posibilita el reconocimiento de las diversas realidades, para los académicos, del área de la salud, insertados en programas de internacionalización posibilita la ejecución del conocimiento adquirido en la realidad actual del futuro profesional y del Brasil. Además de la inserción a una nueva cultura el intercambio posibilita una experiencia con nuevos métodos de enseñanza, nuevas formas de actuación profesional y diferentes modelos de asistencia a la salud. ${ }^{2}$

En la realidad de la enfermería, el intercambio internacional además de promover el contacto del alumnado con modelos de enseñanza ofrece la posibilidad de actuación en modelos asistenciales de salud diferentes de nuestro Sistema Único de Salud. Configurando una experiencia muy enriquecedora, posibilita el intercambio de saberes, añade nuevos valores y calificación del futuro profesional, siendo capaz de provocar cambios progresivos en la reflexión y en la actuación diaria de los enfermeros asistenciales. ${ }^{6}$

Ante lo expuesto, este trabajo tiene como objetivo relatar la experiencia de la movilidad académica internacional en México a través del Programa Internacional de Estudiantes
Brasil-México, durante la graduación de enfermería.

\section{METODOLOGÍA}

Se trata de un relato de experiencia sobre la vivencia y las actividades realizadas durante la ocurrencia del intercambio académico en México por una estudiante de enfermería de la universidad Federal de Mato Grosso en la Universidad Juárez del Estado de Durango (UJED) Facultad de Enfermería y Obstetricia (FAEO), convocada por el Programa Internacional de Estudiantes BrasilMéxico, en el semestre $B$, de agosto a diciembre de 2017.

La selección de los contenidos curriculares de la FAEO para la construcción del plan de estudios fue elegidos de modo a contemplar el servicio social hospitalario desarrollado en el cuarto año del curso de Bachillerato en Enfermería en Brasil. En México, el servicio social de enfermería es desarrollado por medio del Programa de Servicio Social de Pasantes de Enfermería.

La FAEO realizó los trámites y viabilizó la realización del estadio hospitalario curricular de enfermería en el Hospital General de Durango Materno Infantil, posibilitando la experiencia de la actuación profesional de la enfermería mexicana y que culminaron con la construcción de este relato. La etapa totalizó 660 horas, con carga horaria semanal de 40 horas, pasando por los respectivos sectores: Habitación Conjunta, Clínica de Catéteres, Pediatría, Urgencias Pediátricas, Unidad de Terapia Intensiva Neonatal y Unidad de Terapia Intensiva Pediátrica. 


\section{NURSING \\ ANO HEALTH}

\section{RESULTADOS}

\section{Los trámites del intercambio}

Considerando la relevancia profesional y el bagaje de conocimiento que un intercambio posibilita, la decisión de elección por participar del programa de internacionalización comprende en un gran desafío. El contacto con una nueva cultura, convivir con otro idioma, vivir en otro país con hábitos, moneda y costumbres diferentes hace que la inmersión a lo desconocido sea una experiencia desafiante.

La decisión de participar en el programa se sucedió durante el penúltimo semestre del curso de enfermería. Por medio de la Secretaría de Relaciones Internacionales de la UFMT, que lanzó un edicto interno de movilidad internacional, que exija el cumplimiento de los requisitos necesarios para participar de la selección a las universidades extranjeras.

Las instituciones extranjeras establecieron criterios específicos e independientes para las presentaciones de las candidaturas de los estudiantes. Entre las exigencias necesarias en el edicto, obtener nivelación en la prueba de español exigió esfuerzo y compromiso, así como atender a las demandas del proceso de inscripción en el programa.

Se realizó una investigación sobre las instituciones extranjeras, se determinó sobre las asignaturas ofrecidas, los requisitos mínimos para el ingreso y la infraestructura ofrecida por las instituciones, generando un interés por la FAEO de Durango. Capital
ISSN 2236 - 1987

del estado con una población de 565.300 habitantes, situada en la región norte de México.

\section{Las vivencias en Durango}

Fundada hace 455 años, es posible caminar por la ciudad de Durango y encontrar bellas construcciones de la era colonial, una verdadera resistencia cultural. Con la mezcla de las culturas indígenas y españolas, expresada en formas de música, danza, artesanías, costumbres y tradiciones. En el último semestre del año existen muchas celebraciones importantes para los mexicanos, una de ellas es la conmemoración del día de la independencia mexicana, donde es posible observar el amor por la patria y el respeto por las conquistas mexicanas. Toda la ciudad se ilumina con los colores de México, en las casas es posible ver banderas valladas, en el hospital decoraciones con los colores de la bandera y los rostros de los revolucionarios. En las facultades todos se reúnen para comer comidas típicas duranguense y dar nuevamente el grito de la independencia.

Otra tradición encantadora de México es la celebración del Día de Los Muertos, el primero de noviembre se conmemora el "Día de todos los Santos" dedicado a los difuntos niños y el día dos se festeja a los difuntos adultos. Los familiares construyen altares para los muertos, con sus ofrendas, como alimentos que el difunto más le gustaba durante la vida, con velas y flores, para que cuando vengan a visitar puedan revivir y sentirse con cuando tenían vida. Fue posible visitar al panteón en el día de la celebración, ver sus tumbas con esculturas, ver 


\section{JOURNALF

muchas familias visitando a sus entes queridos con música y comida, cambiando mi perspectiva sobre la muerte.

La Coordinación de Internacionalización y Cooperación Académica (CICA) fue un gran socio durante toda la movilidad, convocando a un estudiante para ser anfitrión y ayudar en la recepción. Mi anfitriona fue la primera persona que tuve contacto en Durango, me acogió en la carretera y me llevó al hostel, después me ayudó a encontrar una casa. El anfitrión es un soporte importante en el intercambio, principalmente por haber realizado una movilidad, conoce las necesidades del extranjero. La CICA promovió encuentros entre los demás estudiantes de movilidad, permitiendo una interacción con estudiantes de otras partes de México y del mundo, sin contar vínculos creados.

En el Hospital mi recepción no fue diferente, todos los sectores en que pasé fui muy bien recibida, cada equipo con su particularidad permitió la creación de un vínculo con los profesionales y la transferencia de saberes. Muchas enfermeras fueron como mamas, me cuidando enseñándome a comer las comidas picantes y un poco más sobre la cultura mexicana, personas que admiro personal y profesionalmente. Los profesionales tenían curiosidad sobre la cultura brasileña, sobre las comidas, las ropas, los indígenas, la calidad de vida, entre otras cosas. Lo que más me marcó en aquellos días era el calor humano que me hacía sentir en casa, incluso a más de ocho mil kilómetros.
Las actividades desarrolladas en la Facultad de Enfermería y Obstetricia

La FAEO trabaja en la formación de profesionales de enfermería, formando técnicos de enfermería, licenciados en enfermería y cuenta con cursos de especialización para los profesionales de enfermería. En México, durante la graduación los alumnos realizan sus disciplinas teóricas y prácticas durante los ocho primeros semestres.

Al finalizar las responsabilidades académicas celebran el fin de la graduación, pero no se consideran titulados en enfermería. Pasan por el proceso probatorio que consiste en la realización del Servicio Social, que corresponde al estadio curricular desarrollado durante la graduación de enfermería en Brasil, denominados pasantes de enfermería. Al finalizar esa etapa reciben el título.

Según las Normas oficiales Mexicanas, que se refieren a las directrices sobre el Programa de Servicio Social de Pasantes de Enfermería, los pasantes deben cumplir las siguientes actividades contenidas en dos programas: El programa académico, elaborado por la institución de enseñanza, con las actividades que van a colaborar en el proceso de enseñanza y aprendizaje; $\mathrm{El}$ programa operativo que es elaborado por la institución de salud, basado en el programa académico, con las actividades que los pasantes deben desarrollar en las instituciones de salud.

La FAEO posibilitó la realización del Servicio Social en el sector 
hospitalario, entre las opciones ofrecidas, opté por el hospital que ofrecía especialidad en cuidados Materno e Infantil. El ciclo de prácticas del servicio social se inició en agosto y se concluyó en diciembre de 2017, equivalente a un semestre lectivo. $\mathrm{El}$ servicio social fue desarrollado en el Hospital General de Durango Materno Infantil, en los sectores de habitación conjunta, clínica de catéteres, pediatría, urgencia pediátrica, unidad de terapia intensiva neonatal y unidad de terapia intensiva pediátrica.

\section{El servicio social de enfermería}

Los pasantes de enfermería son una clase de no profesionales de enfermería subordinada a los profesionales de enfermería. Las actividades desarrolladas durante el servicio social se realizan bajo la supervisión de los profesionales de enfermería responsables de los sectores hospitalarios. La propuesta del servicio social, así como el curso curricular, de perfeccionar los conocimientos que se adquirieron durante la graduación. Se convierte en un momento importante en la formación del enfermero permitiendo que el alumnado se desarrolle con autonomía e intensificando la unión de conocimientos teóricos y prácticos.

Importe resaltar sobre las diferencias entre las categorías profesionales de enfermería, en Brasil la profesión es ejercida por el equipo de enfermería, compuesta por el enfermero y técnicos de enfermería. Cada uno de estos profesionales ejercen funciones diferentes dentro del equipo de enfermería, pero complementándose. En México, la práctica de enfermería es ejercida de forma igual por el personal de enfermería, siendo: profesional de enfermería, licenciado en enfermería, enfermero especialista, enfermero maestro, enfermero doctor, profesional técnico de enfermería, técnico de enfermería y técnico de enfermería especializado. $Y$ subordinado a esos profesionales están los no profesionales de enfermería, como auxiliares, estudiantes 0 pasantes de enfermería.

La práctica desarrollada por el profesional de enfermería en México se concentra en la gestión de los cuidados y en la asistencia total al paciente. Las actividades realizadas durante el servicio social se enfocan en la implementación de la asistencia a los pacientes, contemplando las responsabilidades que el profesional debe desempeñar.

Además de la oportunidad de desarrollar el servicio social en el sector hospitalario, la experiencia del intercambio también propició la interacción con la comunidad de la ciudad de Durango por medio de la asistencia de enfermería realizada en el hospital. En el inicio presenté dificultad en la comunicación con mis pacientes y sus familiares, era posible percibir la falta de dominio de la lengua y después de explicar el motivo por tener un acento diferente venia la sorpresa por ver a un extranjero trabajando en el hospital. Para superar ese desafío yo siempre repetía con las enfermeras las frases de las orientaciones para que fuera clara la comprensión y poco a poco la comunicación dejó de ser un problema. Sin contar el intercambio de saberes y 


\section{ISSN 2236 - 1987}

cultura entre el estudiante de intercambio y el equipo de profesionales del hospital. De esta forma, el servicio social generó un impacto positivo en la construcción del futuro profesional $y$ de los profesionales mexicanos que tuvieron la oportunidad de conocer un poco sobre la cultura y la enfermería brasileña.

Aunque la estrategia pedagógica del servicio social es similar a la etapa curricular en Brasil, el Programa de Servicio Social de Pasantes de Enfermería se destaca por la metodología, con una carga horaria mayor a ser cumplida lo que posibilita un mayor aprendizaje práctico. Esta estrategia es priorizada con el objetivo de capacitar a los profesionales en su autonomía, ya que en la graduación las disciplinas prácticas los estudiantes tienen como preceptor a los enfermeros del hospital y no asumen toda la asistencia de los pacientes. A diferencia de las disciplinas prácticas que realice durante la graduación, en que el profesor acompaña en campo práctico y el estudiante asume todos los cuidados asistencias de dos o tres pacientes. Es importante resaltar que otros pasantes del programa comentaron sobre el miedo a ser responsable de todo el asistencialismo a ser desarrollado a los pacientes.

\section{DISCUSIÓN}

El mundo globalizado contribuye en la remoción de fronteras, permitiendo la interacción entre diversas realidades y posibilitando intercambios de cultura y saberes. Pero la globalización también es responsable del intenso flujo de personas, mercancías y enfermedades transmitidas entre los países. Aumentando los riesgos para la salud presentando consecuencias globales, impulsando el interés en investigaciones sobre la salud global y los determinantes que las influencian. Considerando todo este impacto, la necesidad de conocer nuevos sistemas de salud en la práctica configura un método valioso para el crecimiento de la mirada respecto a las políticas públicas de salud. ${ }^{1,4}$

El intercambio permite el enriquecimiento personal debido al desarrollo de la capacidad de adaptación a las diversas situaciones, como el nuevo idioma, nuevos hábitos de vida, moneda y cultura. En la construcción del individuo como profesional, permite el contacto con un sistema de salud diferente $y$ experiencia la práctica de enfermeros de otro país, lo que posibilita una reflexión sobre todas esas diferencias. $^{3-4} \quad$ Este conjunto contribuye a la formación del pensamiento crítico, la valorización y el respeto con su propia cultura e impulsa una movilización interna para cambiar su realidad.

Es importante resaltar que la movilidad internacional durante la graduación causa una diferencia significativa en la construcción del profesional de enfermería. El estudiante de intercambio es el actor principal de este curso permitiendo la amplitud de la capacidad de autonomía e independencia personal. En movilidad internacional la mayor barrera puede ser considerada la inhabilidad con la lengua, por dificultar la interacción social 
convirtiéndose en el primer desafío a ser enfrentado. La habilitación en otro idioma, a través de la profesión, contribuye a la cualificación curricular de los profesionales del área de enfermería, teniendo en cuenta que las actualizaciones científicas con divulgadas por medio de artículos en inglés y español. 3-4,6-7

\section{CONSIDERACIONES FINALES}

La movilidad internacional durante la graduación de Enfermería entre posibilitó la vivencia de experiencias con significancia en los aspectos personales y profesionales. Realizar una etapa durante la movilidad es una experiencia enriquecedora, posibilitando la oportunidad de conocer estructuras, rutinas e sistemas de salud diferente de lo habitual. Sin contar la convivencia con el idioma, la cultura, los hábitos para agregar valores al crecimiento profesional y personal.

Las diferencias de la realidad brasileña y mexicana tienen el reflejo en el área del cuidado y el intercambio posibilitó reflexiones sobre las diferencias de la actuación del enfermero posibilitando ampliar los conocimientos sobre la enfermería. Con respecto a toda esa diferencia categórica de los profesionales de enfermería fue posible despertar interés en conocer sobre la variación de la actuación profesional de la enfermería en el mundo.

En vista de lo expuesto, la movilidad internacional puede ser considerada una importante herramienta de instrucción para la formación de enfermeros, ya que estimula la maduración profesional y personal de los estudiantes, por la superación de desafíos. Experiencia que refleja sobre la actuación práctica del estudiante y los conocimientos adquiridos. Contribuyendo al estímulo de la búsqueda por el saber y mejorando la asistencia de enfermería dentro de las diversidades.

\section{REFERENCIAS}

1 Carvalho JL, Backes DS, Lomba MLLF, Colome JS. Intercâmbio académico internacional: uma oportunidade para a formação do futuro enfermeiro. Rev enf ref [Internet]. 2016 jul/ago[acceso em 2018 ago 13];4(10):59-66. Disponible:

http: / /www.scielo.mec.pt/pdf/ref/vs erIVn10/serIVn10a07.pdf

2 Patuzzi GC, Lira APS, Santos JDM, Reinisch AOB, Veleda AA. Academic learning about public health in a Canadian university: contributions for Brazilian education. Rev gauch enferm [Internet]. 2017 Mar[cited 2018 Aug 13];38(3):e2016-0012. Available from: http: / / www.scielo.br/pdf/rgenf/v38n 3/en_1983-1447-rgenf-198314472017032016-0012.pdf

3 Guedes GF, Reis GT, Coelho S, Oliveira M De, Reis CC. Intercâmbio internacional nos cursos de graduação de enfermagem. J nurs health [Internet]. 2017[acesso em 2018 ago 13];7(2):213-25. Disponível em: https://periodicos.ufpel.edu.br/ojs2/ index.php/enfermagem/article/view/ $11009 / 7890$

4 Ferreira IG, Carreira LB, Botelho NM. Mobilidade internacional na graduação em medicina: relato de experiência. ABCS health sci [Internet]. 2017[acesso em 2018 ago 13];42(2):115-9. 


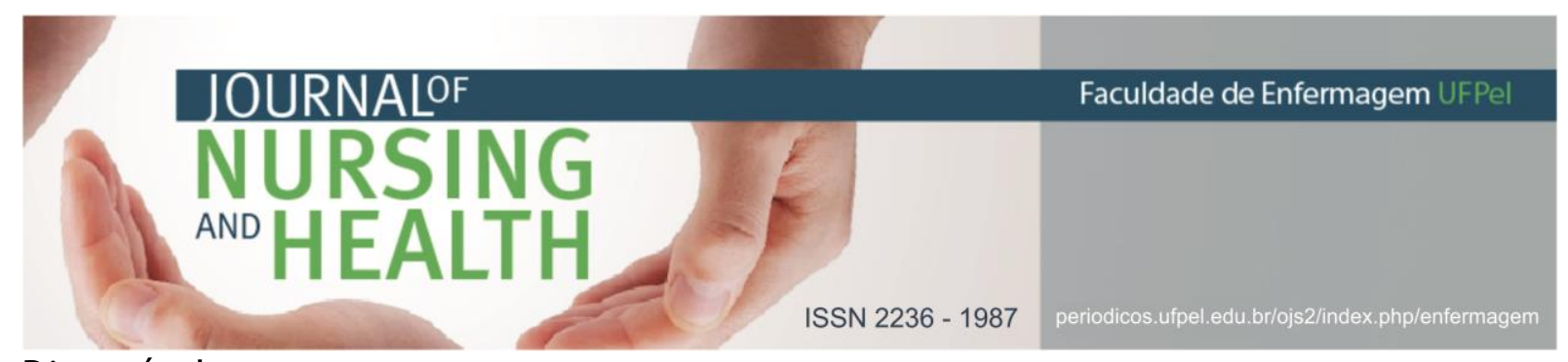

Disponível em: https://www.portalnepas.org.br/abcs hs/article/view/1013/776

5 Jung BC, Freitag VL, Gonzáles RIC, Dalmolin IS. Colômbia: cenário de estudo em enfermagem no programa de mobilidade acadêmica internacional. Rev enferm UFSM [Internet]. 2015 out/dez[acesso em 2018 ago 13];5(4):675-82. Disponível em:

https: / / periodicos.ufsm.br/reufsm/ar ticle/view/18952/pdf

6 Guskuma EM, Dullius AAS, Godinho MLSC, Costa MST, Terra FS. Mobilidade acadêmica internacional na formação em enfermagem: relato de experiência. Rev bras enferm [Internet]. 2016 set/out[acesso em 2018 ago 13];69(5):986-90. Disponível em:

http://www.scielo.br/pdf/reben/v69 n5/0034-7167-reben-69-05-0986.pdf

7 Andrade DMB, Boery RNSO. Revealing the borders of knowledge by the international academic. Rev enferm UFPE online [Internet]. 2016[cited 2018 Aug 13];10 Suppl.S:4951-8. Available from: https://periodicos.ufpe.br/revistas/r evistaenfermagem/article/view/1127 7/12919

Data de submissão: 28/05/2018

Data de aceite: 27/06/2018

Data de publicação: 15/10/2018 